\title{
Solvatochromic and fluorogenic dyes as environment-sensitive probes: design and biological applications
}

\author{
Andrey S. Klymchenko \\ Laboratoire de Biophotonique et Pharmacologie UMR 7213 CNRS/Université de Strasbourg, 67000 \\ Strasbourg, France.E-mail: andrey.klymchenko@unistra.fr
}

\section{Conspectus}

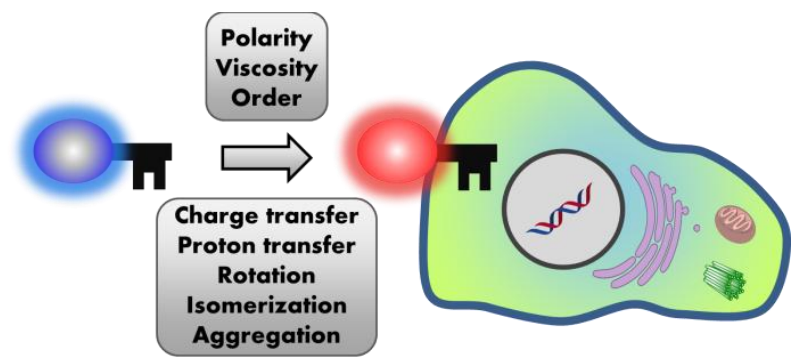

Fluorescent environment-sensitive probes are specially designed dyes that change their fluorescence intensity (fluorogenic dyes) or color (chromogenic dyes) in response to change in their microenvironment polarity, viscosity and molecular order. The studies of the last decade, including those of our group, have shown that these molecules become universal tools in fluorescence sensing and imaging. In fact, any biomolecular interaction or change in biomolecular organization results in modification of the local microenvironment, which can be directly monitored by these types of probes. In this Account, the main examples of environment-sensitive probes are summarized according to their design concepts. The majority of environment-sensitive chromogenic probes are solvatochromic dyes which change their color in response to polarity. Generally, they are push-pull dyes undergoing intramolecular charge transfer. Excited-state intramolecular proton transfer is the second key concept to design efficient solvatochromic dyes. Due to sensitivity to polarity and hydration, solvatochromic dyes have been successfully applied to biological membranes for studying lipid domains (rafts), apoptosis and endocytosis. As fluorescent labels, solvatochromic dyes can detect practically any type of biomolecular interactions, involving proteins, nucleic acids and biomembranes, because the binding event excludes local water molecules from the interaction site. On the other hand, fluorogenic probes usually exploit intramolecular rotation (conformation change) as a design concept, with molecular rotors being main representatives. These probes were particularly efficient for imaging viscosity and lipid order in biomembranes as well as to light up biomolecular targets, such as antibodies, aptamers and receptors. The emerging concepts to achieve fluorogenic response to the microenvironment include ground-state isomerization, aggregation-caused quenching and aggregation-induced emission. The ground-state isomerization exploits, for instance, polarity-dependent spiro-lactone formation in silica-rhodamines. The aggregation-caused quenching uses disruption of the self-quenched dimers and nano-assemblies of dyes in less polar environments of lipid membranes and biomolecules. The aggregation-induced emission couples target recognition with formation of highly fluorescent dye aggregates. Overall, the environmentsensitive fluorogenic and chromogenic probes enable background-free bioimaging in wash-free conditions as well as quantitative analysis when combined with advanced microscopy, such as fluorescence lifetime (FLIM) and ratiometric imaging. Further development of fluorescent environment-sensitive probes should address some remaining problems: (i) improving optical properties, especially brightness, photostability and far-red to near-infrared operating range; (ii) minimizing non-specific interactions of the probes in biological media; (iii) their adaptation for advanced microscopies, notably for superresolution and in vivo imaging. 


\section{Introduction}

Fluorescence is one of the most popular among existing biosensing and bioimaging techniques, because it allows investigation of biological samples directly in situ and provides rich information at the biomolecular level with high spatial and temporal resolution. As the biological objects are poorly fluorescent, the current fluorescence techniques rely strongly on fluorescent dyes, proteins and nanoparticles. Fluorescent dyes are of particular importance because of their small size, possibility to finely tune their properties and easiness of chemical modification. Dyes that are specially designed to perform biosensing or bioimaging tasks are called fluorescent molecular probes. Some probes can specifically target biomolecules, cellular organelles, etc, whereas others enable detection and imaging of metal ions, $\mathrm{pH}$, etc. In this Account, we focus on probes that change their emission characteristics in response to fundamental properties of molecular environment: polarity, viscosity and molecular order. These probes have become universal tools in fluorescence sensing and imaging, because any biomolecular interaction or change in biomolecular organization results in site-specific modification of these properties. Based on the response type, there are fluorogenic and chromogenic probes, which change their fluorescence intensity and emission color, respectively. In this Account, the main examples of environment-sensitive probes and their applications are summarized according to mechanism of their operation (Fig. 1). We should note that other types of fluorogenic and chromogenic probes, which respond to ions, ${ }^{1} \mathrm{pH},{ }^{2}$ bioconjugation reactions, ${ }^{3}$ specific cellular organelles, ${ }^{4}$ etc, ${ }^{5}$ have been well reviewed and they are out of the scope of this Account.
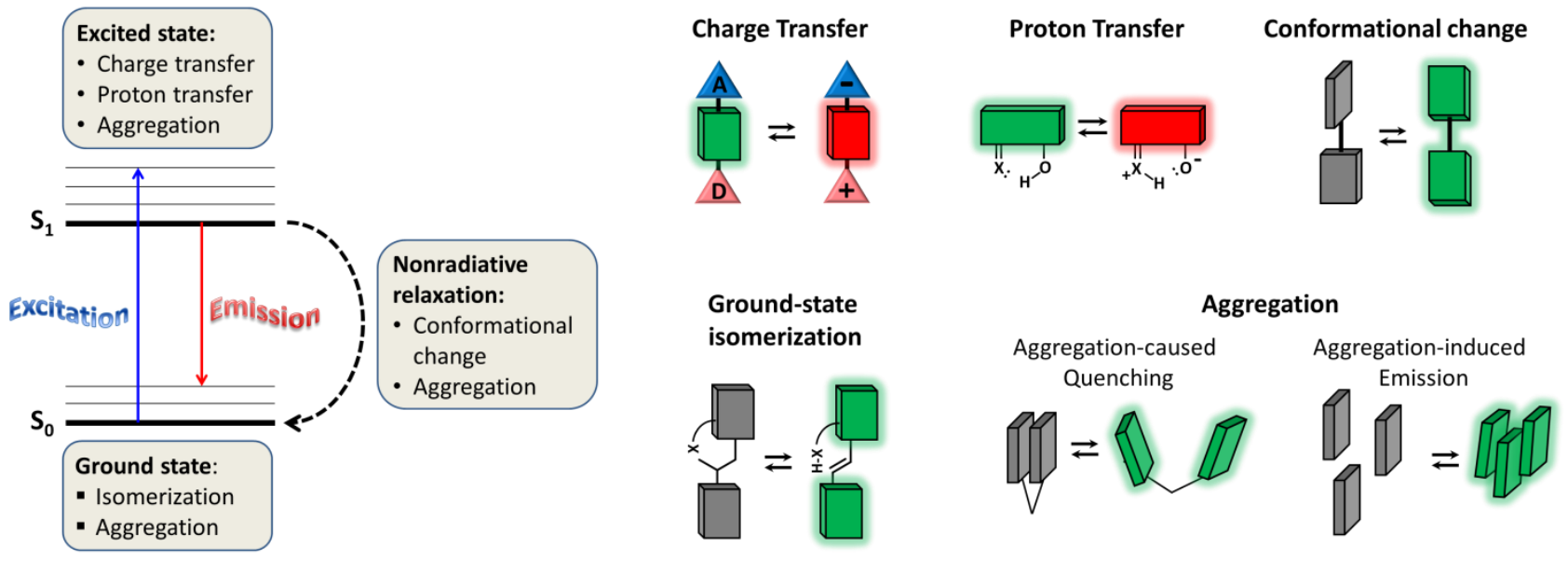

Fig. 1. Simplified Jablonski diagram and the key mechanisms involved in the environment-sensitive probes.

\section{Probes based on charge transfer}

The classical environment-sensitive dyes are aromatic structures presenting donor and acceptor groups, so-called push-pull fluorophores. The push-pull structure ensures that after light absorption the charge is transferred from the donor group to the acceptor, which creates a highly dipolar excited state (Fig. 2a). The latter relaxes through interaction with the dipoles of polar solvents and thus emits at longer wavelengths (Fig. 2a,b). A variety of the pushpull dyes are present in the literature, notably Prodan, Dansyl, 4DMP, NBD, Nile Red, Dapoxyl derivatives, etc (Fig. 2a). Until recently, the dyes with the strongest solvent-dependent red shifts were Fluoroprobe, Dapoxyl and Prodan, all absorbing in the UV region (Fig. 2c). In our previous work, we developed fluorene analogue of Prodan, FR0, featuring extended conjugation (Fig. 2a). ${ }^{6}$ In addition to absorption shifted to $400 \mathrm{~nm}$, the new dye showed higher extinction coefficient (43000 vs $18400 \mathrm{M}^{-1} \mathrm{~cm}^{-1}$ ), two-photon absorption cross-section (400 vs $75 \mathrm{GM}$ ), quantum yield and photostability. Moreover, FR0 exhibited improved sensitivity to polarity (spectral shift of $5500 \mathrm{~cm}^{-1} \mathrm{vs}$ $3900 \mathrm{~cm}^{-1}$ of Prodan, Fig. 1b,c) due to its high transition dipole moment (14 D). The use of pyrene as aromatic moiety also significantly improved properties with respect to Prodan, in particular quantum yield $(\sim 1.0)^{7}$ and 
photostability. ${ }^{8}$ Twieg et al introduced DCDHF family (Fig. 2a), ${ }^{9}$ which is a rare example of solvatochromic dyes operating in the red region, although sensitivity of these dyes to polarity remains close to Nile Red (Fig. 2c). Recent works on push-pull dyes revealed two tendencies. First, photostability of solvatochromic dyes is relatively poor, especially in apolar aprotic solvents, which favor triplet state formation and thus photo-oxidation. ${ }^{6,8}$ Second, most of push-pull dyes are quenched in polar protic solvents, generally due to electron transfer and twisted intramolecular charge transfer (TICT). ${ }^{10}$

(a)

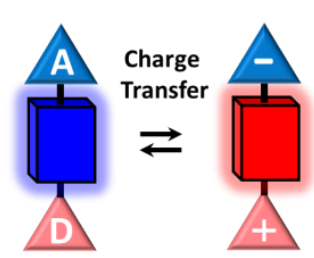

FRO

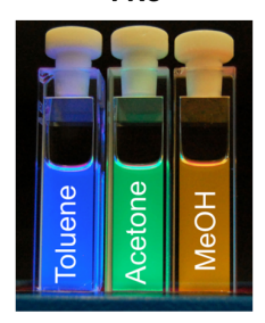

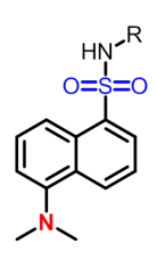

Dansyl

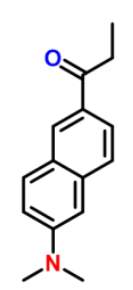

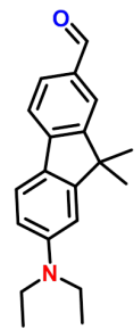

FRO

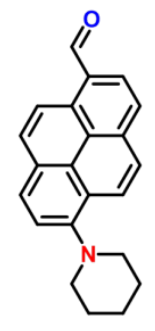

PA

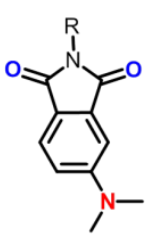

4DMP

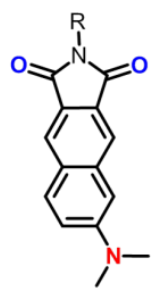

6DMN
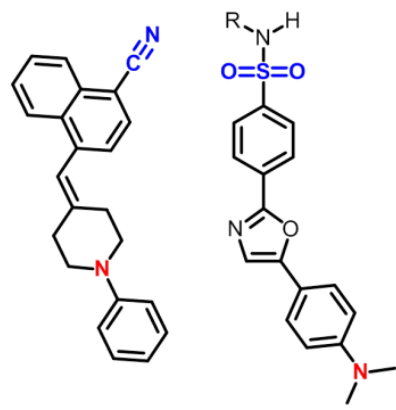

Dapoxyl

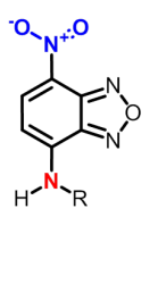

NBD

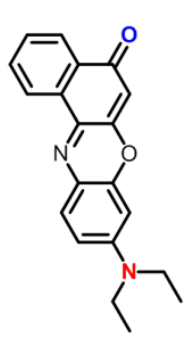

Nile Red

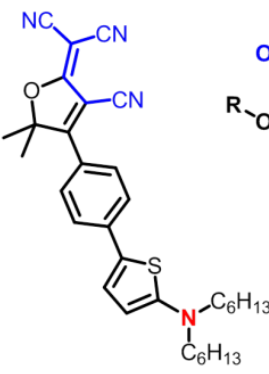

DCDHF-6

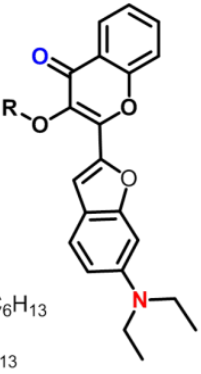

3MC-2 (b)

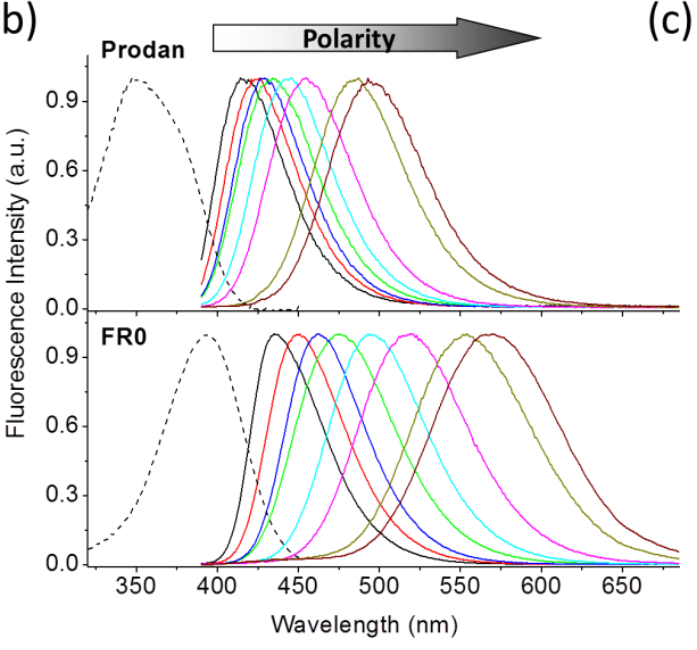

(c)

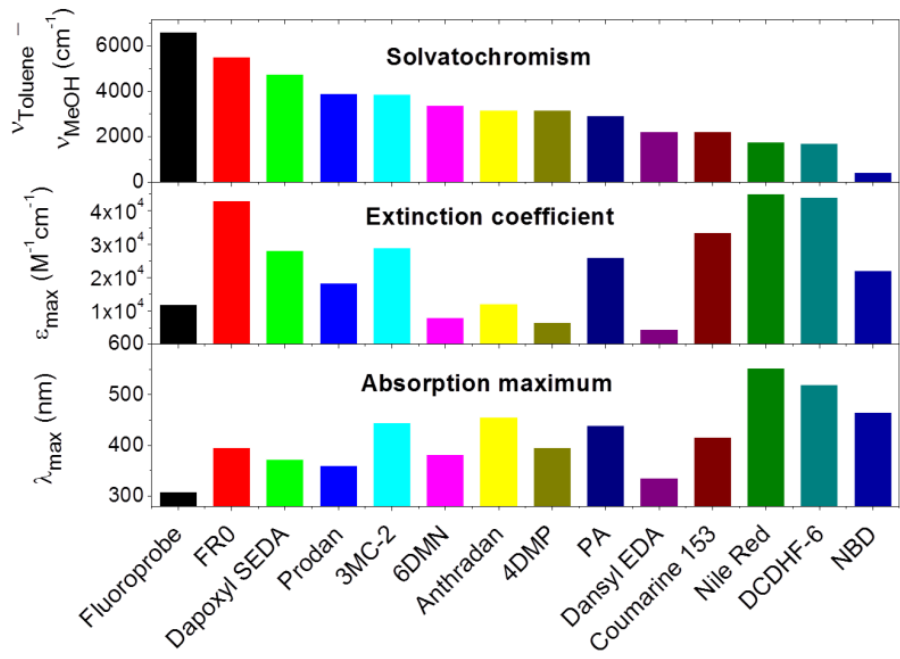

Fig. 2. Push-pull solvatochromic dyes and comparison of their spectroscopic properties. (a) Concept and examples of dyes undergoing excited-state charge transfer. Photo of cuvettes of FR0 in solvents of different polarity. (b) Comparison of polarity-dependent band shifts of Prodan and FR0. Reproduced with permission from ref 6. Copyright 2010 American Chemical Society. (c) Comparison of spectroscopic properties of different solvatochromic fluorescent dyes. $v_{\text {Toluene }}-v_{\mathrm{MeOH}}$ is the shift of the fluorescence maximum from toluene to methanol.

Due to sensitivity to polarity, push-pull dyes enable monitoring intrinsic biophysical properties of biomembranes, such as polarity, hydration, electrostatics, etc. ${ }^{11}$ They are particularly useful for imaging liquid ordered (Lo) and disordered (Ld) phase domains, which, according to hypothesis of lipid rafts, ${ }^{12}$ are believed to play important role in 
organization and functions of biological membranes. Due to their small size and transient nature, lipid rafts are difficult to detect in living cells. Hence, development of advanced fluorescent probes distinguishing Lo and Ld phases is currently in progress. ${ }^{13}$ Lo phase, tightly packed with saturated lipids and cholesterol, presents much lower hydration and polarity as compared to loosely packed Ld phase, composed of unsaturated lipids. ${ }^{11}$ Therefore, solvatochromic dye Laurdan (a lipophilic analogue of Prodan) showed blue shifted emission in the Lo phase compared to the Ld phase. ${ }^{14}$ In our recent work, a red-shifted analogue of Laurdan, push-pull pyrene PA (Fig. 2a), showed similar color response to phase domains, whereas in living cells it revealed a gradual decrease in the lipid order from plasma membrane towards endoplasmic reticulum. ${ }^{8}$ To label specifically the outer plasma membrane leaflet, where the key component of Lo phase, sphingomyelin, is mainly present, Loew et al introduced push-pull probe di-4-ANEPPDHQ, bearing both alkyl chains and charged groups (Fig 3a). ${ }^{15}$ In our studies, we developed probe NR12S based on Nile Red, bearing dodecyl chain and zwitterion group (Fig. 3a). ${ }^{16}$ Unlike Nile Red, NR12S binds exclusively the outer membrane leaflet of biomembranes with negligible flip-flop on the time scale of hours. NR12S enabled ratiometric imaging of Lo and Ld phase domains in model membranes (Fig. 3b,c) and cholesterol depletion in cell membranes (Fig. 3d). ${ }^{16}$ In comparison to di-4-ANEPPDHQ, NR12S requires much lower concentration for cell membrane imaging $(20-50 \mathrm{nM}$ vs $1-5 \mu \mathrm{M})$, although photostability of NR12S is limited. ${ }^{17}$ Numerous applications of NR12S include imaging maturation of endosomes, ${ }^{18}$ conformational changes of membrane proteins, ${ }^{19}$ internalization of nanostructures ${ }^{20}$ and detection of apoptosis. ${ }^{17}$ We should note that charge transfer dyes, being vertically oriented in biomembranes, can also sense electric fields, notably transmembrane potential, as shown by Loew et al for styryl-pyridinium dyes, ${ }^{21}$ analogues of di-4-ANEPPDHQ.

(a)

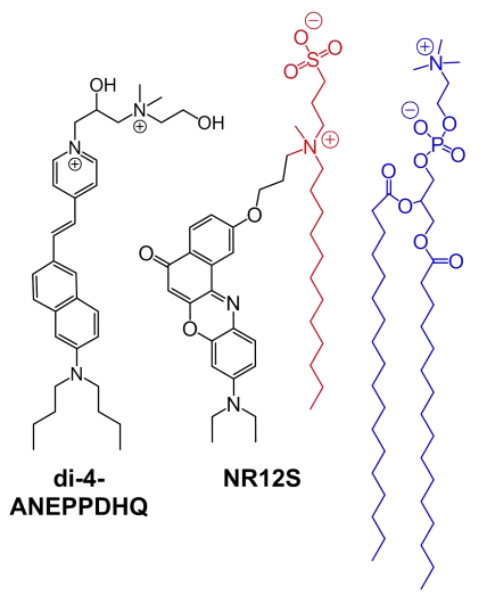

(b)

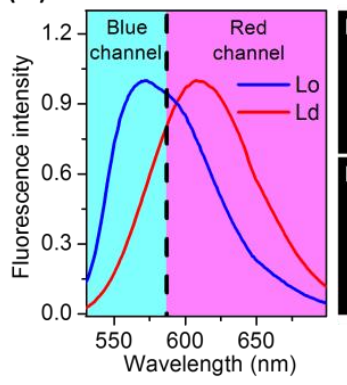

(c)

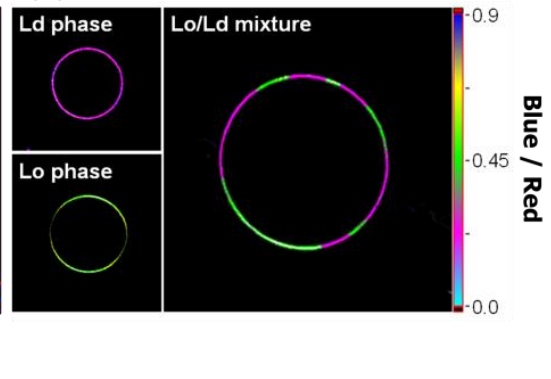

(d)
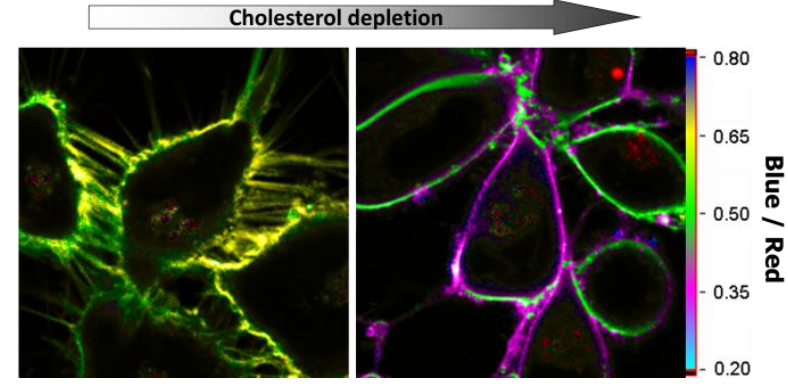

Fig. 3. Applications of push-pull probe NR12S for probing lipid order in biomembranes. (a) Examples of leafletspecific probes for lipid rafts. (b) Fluorescence spectra of NR12S in lipid vesicles presenting Lo and Ld phases. (c) Ratiometric images of giant vesicles presenting Lo and Ld phases and their mixture. (d) Decrease in the lipid order in cell membranes (U87MG human glioblastoma) on cholesterol depletion by methyl- $\beta$-cyclodextrin followed by ratiometric imaging with NR12S probe. Adapted with permission from ref ${ }^{16}$. Copyright 2010 American Chemical Society.

The second important application of solvatochromic probes is detection of biomolecular interactions, as a powerful alternative to traditional methods based on Forster Resonance Energy Transfer (FRET) and fluorescence anisotropy. ${ }^{22}$ In fact, interaction of biomolecular partners should basically result in the exclusion of the local water 
molecules, thus decreasing the environment polarity. The early examples of push-pull dyes used to monitor interactions of biomolecules included amino acid analogues of Prodan ${ }^{23}$ and $4 \mathrm{DMN},{ }^{24}$ operating in the ultravioletviolet region. Using Nile Red conjugated to carbetocin through polyethylene glycol (PEG) spacer, we obtained a turn-on probe for wash-free detection of $G$ protein-coupled oxytocin receptor using convenient red spectral region. ${ }^{25}$ PEG8 linker was important to prevent non-specific interaction with serum and lipid membranes.

\section{Probes based on intramolecular proton transfer}

Generally, dyes undergoing excited-state intramolecular proton transfer (ESIPT) are sensitive to the environment only when their intermolecular H-bonding is weakened by rotational freedom and donor/acceptor substituents. ${ }^{26}$ The typical examples are $\mathrm{HBON}$, diCN-HBO and SAN, all presenting solvent-dependent dual emission. ${ }^{26}$

Alternatively, efficient perturbation of $\mathrm{H}$-bonding can be achieved in dyes with 5-membered $\mathrm{H}$-bonding ring, such as 3-hydroxychromone family (3HC; Fig. 4) ${ }^{11,26}$ and its aza-analogues, 3-hydroxyquinolones (3HQ). ${ }^{27}$ Their emission is composed of two well-separated bands of normal $\left(\mathrm{N}^{*}\right)$ and tautomeric $\left(\mathrm{T}^{*}\right)$ states (Fig. 4b). The ratio of intensities $\left(\mathrm{N}^{*} / \mathrm{T}^{*}\right)$ increases with increase in the solvent polarity and $\mathrm{H}$-bond donor ability, because polar and especially protic solvents inhibit ESIPT reaction (Fig. 4c). Remarkably, polarity range, where solvent-dependent dual emission is observed, depends strongly on the substituents. ${ }^{28}$ Thus, dyes $3 \mathrm{HF}$ and $3 \mathrm{HC}$ operate in highly polar environments between water and acetonitrile, whereas DMA-3HF and FA operate in solvents of medium polarity due to strong electron-donor groups enhancing dipole moment of the $\mathrm{N}^{*}$ state (Fig. 4b). ${ }^{29}$

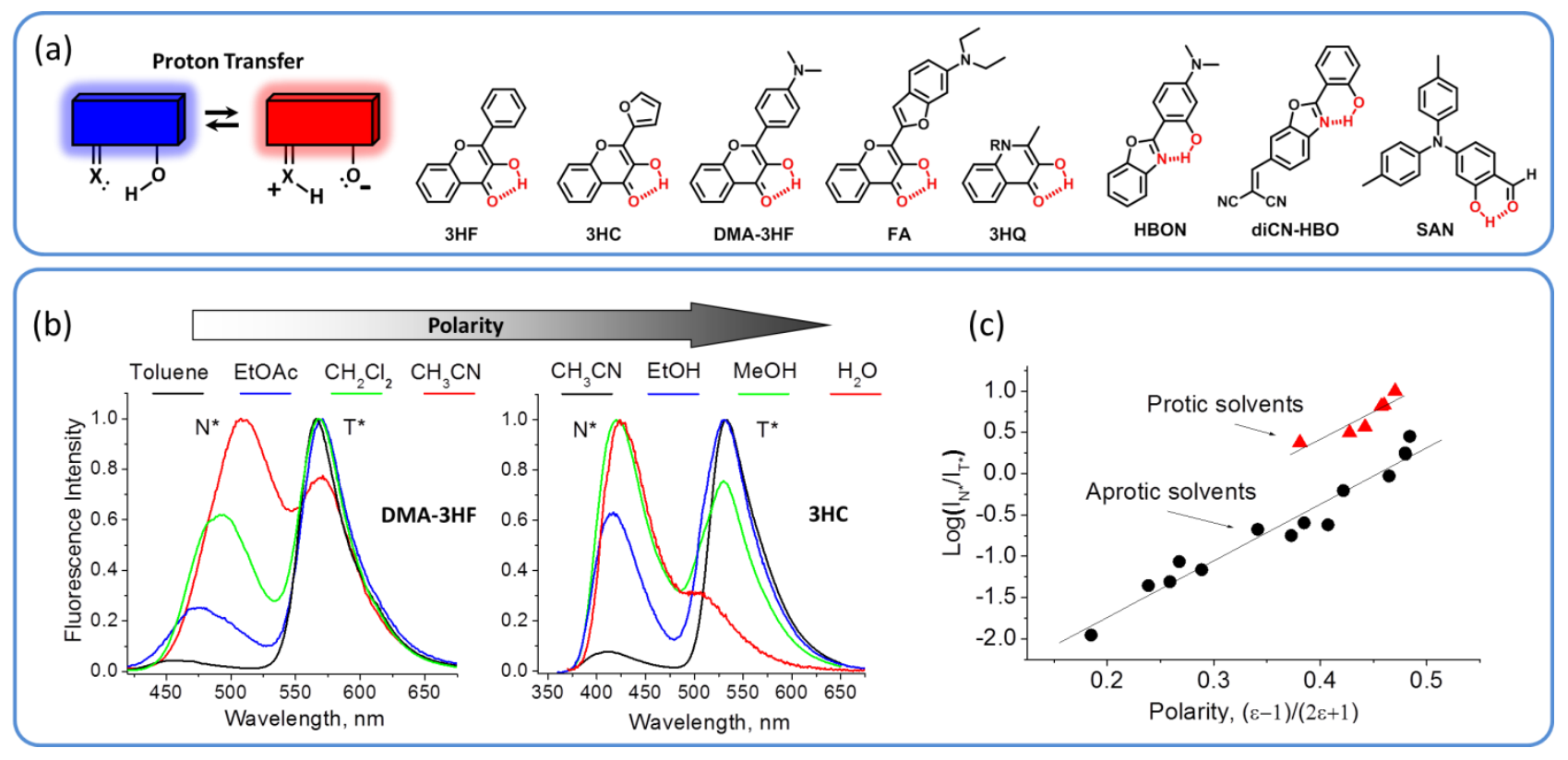

Fig. 4. Fluorescent dyes undergoing ESIPT and displaying solvent-dependent dual emission (a). (b) Fluorescence spectra of 3-hydroxychromone dyes in solvents of different polarity. Adapted with permission from ref ${ }^{22}$. Copyright 2013 Elsevier. (c) Dependence of dual emission from of DMA-3HF on solvent polarity. Adapted with permission from ref ${ }^{29}$. Copyright 2003 PCCP Owner Societies.

3HCs found variety of applications as membrane probes. Dyes vertically oriented in the lipid bilayer are sensitive to dipole (F8N1S and PPZ8) ${ }^{30}$ and transmembrane potentials (di-SFA), ${ }^{31}$ while those with tilted orientation, were more suitable to sense lipid order and surface potential (Fig. 5). ${ }^{11,32}$ In this respect, we should mention F2N12S, which due to specific staining of the outer plasma membrane leaflet, could detect changes in the lipid composition during programmed cell death (apoptosis). ${ }^{33}$ The loss of asymmetry between the two leaflets produced by apoptosis 
increased the negative surface charge and decreased the lipid order at the outer leaflet, which was detected by F2N12S as change in the dual emission (Fig. 5).

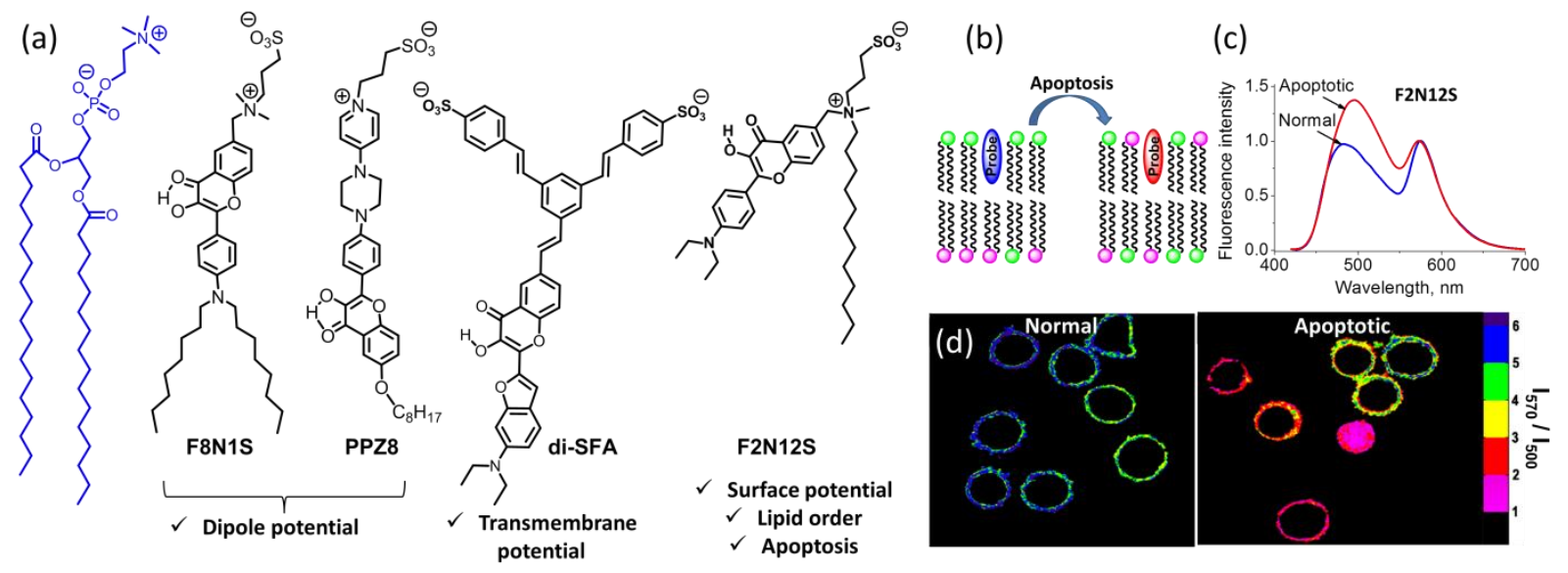

Fig. 5. Application of ESIPT dyes based on 3-hydroxychromone for lipid membranes. (a) Membrane probes based on 3-hydroxychromone. (b-d) Application of F2N12S for apoptosis detection. Schematic presentation of probe in plasma membranes during apoptosis (b) and detection of apoptosis by F2N12S using fluorescence spectroscopy (c) and microscopy (d). Adapted with permission from ref ${ }^{33}$. Copyright 2007 American Chemical Society.

Remarkably, 3HCs has become universal probes for almost any type of biomolecular interactions. ${ }^{22}$ Being grafted to a peptide these dyes could detect the interactions with nucleic acids, ${ }^{34}$ proteins ${ }^{35}$ and lipid membranes ${ }^{36}$ (Fig. 6). The interaction events in all these cases decreased local polarity, which systematically increased relatively intensity of the ESIPT product. 3HC-based fluorescent amino acid (3HC-2) could replace natural ones to probe their insertion into DNA (Fig. 6b). ${ }^{34}$ Nucleotide analogues of 3HCs were applied for detection of DNA hybridization and interaction of chaperon proteins. ${ }^{37}$ Being attached to $\alpha$-synuclein, 3HCs detected the early steps of the peptide aggregation, involved in Parkinson's Disease..$^{38}$ Recently, we applied a specially designed ESIPT dye to study a cell-penetrating peptide penetratin. Ratiometric imaging revealed that penetratin at low concentrations can rapidly enter the cytosol by directly crossing the cell plasma membrane, in addition to a classical endocytosis pathway (Fig. 6d). ${ }^{39}$
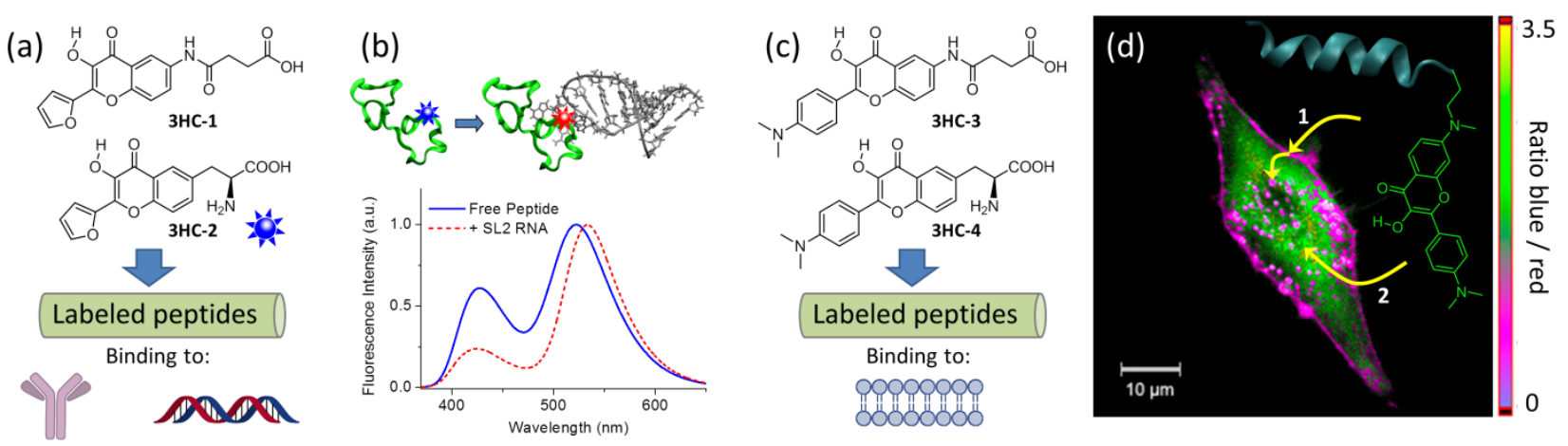

Fig. 6. Sensing and imaging biomolecular interactions by ESIPT dyes of 3HC family. (a) Application of 3HCs for sensing interaction of labeled peptide with nucleic acids and antibodies. (b) Example of application of fluorescent amino acid (3HC-2) for detecting interaction of a peptide with a target oligonucleotide. (c) Application of 3HCs as probes for peptide-biomembrane interaction. (d) Ratiometric imaging of internalization of penetratin into HeLa cells using a 3HC label. Adapted with permission from ref. ${ }^{39}$. Copyright 2014 Royal Society of Chemistry. 


\section{Probes based on conformational changes}

The internal rotation, disrupting the dye planarity, is a source of non-radiative relaxation (Fig. 1), which is used in design of so-called molecular rotors. In viscous environment, where the rotations are slowed down, fluorescence intensity and lifetime of these dyes are strongly enhanced. The classical examples are CCVJ ${ }^{40}$ BODIPY derivative, ${ }^{41}$ Thiofavin $\mathrm{T}^{42}$ and green fluorescent protein (GFP) analogues (Fig. 7a). ${ }^{43,44}$ Generally, molecular rotors are push-pull dyes, which undergo TICT in polar media, resulting in a poorly fluorescent state. ${ }^{10}$ Recently, we introduced a pushpull boron-bridged dye DXB Red,45 which is highly emissive in apolar media, but quenched by TICT in polar solvents. In viscous polar solvent glycerol, its emission is restored, which is typical for molecular rotors. Symmetric non-planar dyes, such as porphyrin dimer ${ }^{46}$ and malachite green (Fig. 7a), ${ }^{47}$ can also function as molecular rotors.
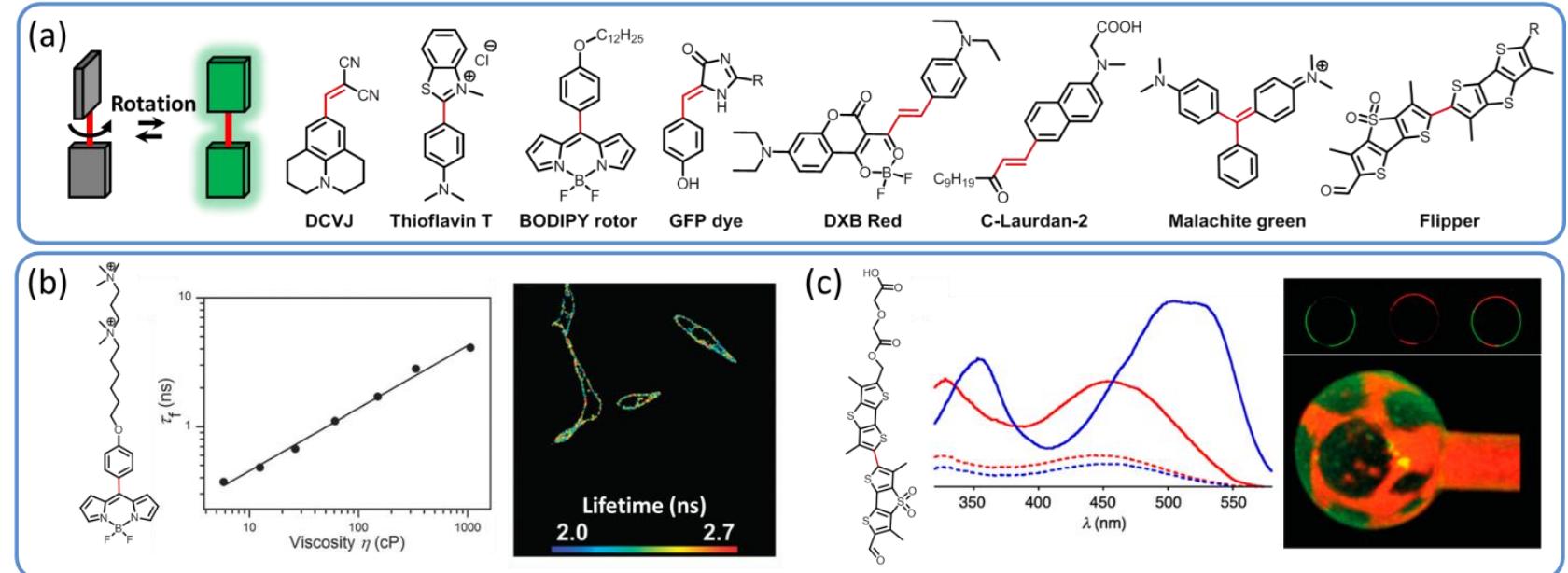

Fig. 7. Probes operating by intramolecular rotation and their applications for membrane research. (a) Concept and examples of the probes. (b) Membrane probe based on BODIPY rotor (left), its fluorescence lifetime vs. viscosity (middle) and FLIM image of SK-OV-3 cells incubated with this probe (right). Adapted with permission from ref. ${ }^{48}$ Copyright 2014 Royal Society of Chemistry. (c) Flipper probe (left), its excitation spectra (middle) in dipalmitoylphosphatidylcholine (DPPC) vesicles (solid) and dioleoylphosphocholine (DOPC) vesicles (dotted) at 25 ${ }^{\circ} \mathrm{C}$ (blue) and $55{ }^{\circ} \mathrm{C}$ (red). Confocal images of giant vesicles containing both Lo and Ld phases stained with flipper probe obtained by excitation at shorter (green vesicle, $\lambda$ ex $=480 \mathrm{~nm}$ ) and longer wavelength (red vesicle, $\lambda$ ex $=560$ $\mathrm{nm})$. Below is $3 \mathrm{D}$ z-stacked image of the same type of vesicle. Adapted with permission from ref. ${ }^{49}$. Copyright 2015 American Chemical Society.

Molecular rotors found variety of applications, especially for probing lipid membranes. One of the earliest examples is FCVJ, a hydrophobic analog of DCVJ (Fig. 7a) developed by Heidekker et al, which was used to visualize shear forces in living cells. ${ }^{40}$ Later on, Kuimova et al proposed BODIPY rotor (Fig. 7a) for fluorescence lifetime imaging (FLIM) of membrane viscosity in living cells. ${ }^{41}$ Its analogue, bearing two cationic groups and long alky chain, enabled viscosity imaging specifically in plasma membranes (Fig. 7b). ${ }^{48}$ Molecular rotors can distinguish higher local viscosity in Lo phase compared to Ld phase, as shown for C-Laurdan-2 (Fig. 7a). ${ }^{50}$ The other application of molecular rotors is background-free imaging of biomolecules. Jaffrey et al developed RNA aptamers that turn on fluorescence of GFP-derived fluorophore (GFP dye, Fig. 7a), ${ }^{43}$ which enabled intracellular RNA imaging. Bruchet et al designed single-chain antibodies that turn-on fluorescence of malachite green, and proposed them for intracellular protein labeling. ${ }^{47}$ Recently, we developed a conjugate of DXB Red with carbetocin that turned on fluorescence on binding to the target oxytocin receptor, due to transfer of the fluorophore from water into low polar viscous environment. It enabled receptor imaging in wash-free conditions with high signal-to-background ratio (130). 45

A new class of probes based on conformation changes, so-called "flippers", was recently introduced by Matile et al. ${ }^{49}$ Due to steric hindrance, flippers present a twisted ground state featuring distorted conjugation between the large 
aromatic units (Fig. 7a). In the rigid environment, such as ordered lipid membranes, the dyes become planar producing the red-shifted absorption. Their excitation spectrum distinguishes well between Lo and Ld phases, while the emission band remains invariant (Fig. 7c). Using excitation at two wavelengths, these probes enabled two-color imaging of phase domains in giant vesicles. Sensitivity of flippers to the mechanical forces in the environment makes them different from solvatochromic dyes and molecular rotors.

\section{Probes based on ground-state isomerization}

Ground state isomerization that breaks the dye conjugation is another important mechanism to achieve fluorogenic response. It is particularly important in rhodamine dyes, where nucleophile carboxy group in the ortho-position of the side phenyl, can attack the electrophilic center of the heterocycle forming spiro-lactone (Fig. 8). Importantly, formation of spiro-lactone rhodamines is a reversible process that depends on the solvent polarity, but it takes place only in apolar media. Recently, Johnsson et al proposed to use silica-rhodamines (SIR), which undergo ring closing at much higher polarity (Fig. 8)..$^{51,52}$ Their conjugates with ligands for tubulin, actin, SNAP tag, etc displayed open fluorescent form only after binding to the target proteins. This fluorogenic behavior enabled multi-color background-free imaging of target proteins in wash-free conditions using conventional and structured illumination microscopy (SIM, Fig. 8). 52

(a)

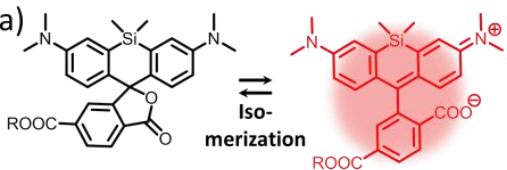

SIR650

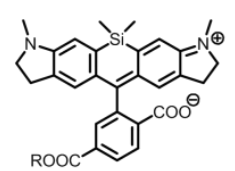

SIR700

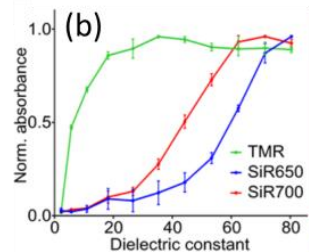

(c)

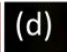

(d)

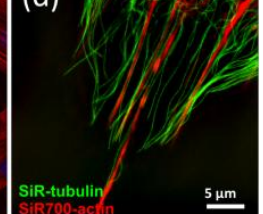

Fig. 8. Probes based on intramolecular isomerization. (a) Concept and examples of the probes. (b) Sensitivity of zwitterion - spiro-lactone equilibrium of tetrametyl rhodamine (TMR), silica-rhodamines to the dielectric constant of solvent. (c) Confocal images of human primary fibroblasts stained with SiR700-actin probe (red) and Hoechst 33342 (blue). (d) Two-color SIM of human primary fibroblasts stained with SiR-tubulin (green) and SiR700-actin (red). Adapted with permission from ref. ${ }^{52}$. Copyright 2016 American Chemical Society.

\section{Probes based on aggregation}

Dyes are flat aromatic structures and, therefore, they have a strong tendency to $\pi$-stack into non-fluorescent $\mathrm{H}$ aggregates. ${ }^{53}$ In water, aggregation-caused quenching (ACQ) is favored by strong hydrophobic interactions, whereas apolar environment can disassemble the aggregates generating a fluorogenic response. The probes can be based on either intramolecular or intermolecular aggregates. The typical example of the former is commercial probe YOYO-1 (Fig. 9a). This dimeric dye is non-fluorescent in water due to H-aggregation, but it opens up on binding to DNA producing fluorescence turn-on. This concept has been applied for sensing interactions with nucleic acids. ${ }^{54}$ Recently, we extended it to polarity sensing. The dimers of squaraine dyes formed poorly fluorescent $\mathrm{H}$-aggregate with characteristic sharp short-wavelength absorption band (Fig. 9b). ${ }^{55}$ Decrease in the polarity produced unfolding of the dimer and thus increase in the fluorescence intensity, especially for dimer with additional PEG groups (Fig. 9c). This design generated polarity-sensitive fluorogenic dyes with unprecedented brightness due to high extinction coefficient $\left(\varepsilon \sim 700,000 \mathrm{M}^{-1} \mathrm{~cm}^{-1}\right)$ and quantum yield $(\sim 0.5)$. Its conjugate with carbetocin ligand showed turn-on response to the target oxytocin receptor in living cells (Fig. 9d), and much lower background in comparison to a 
non-fluorogenic rhodamine-based ligand (Fig. 9e). Moreover, the highest specificity was recorded for the derivative bearing additional PEG groups (Fig. 9f). Recently, another group followed this design concept to further shift the emission to near-infrared for in vivo imaging. 56

Alternative probe design is to exploit disassembly of intermolecular dyes aggregates in water. Hamachi et al, proposed to aggregate variety of dyes, such as rhodamine, fluorescein and BODIPY using a special hydrophobic linker and targeting ligand. ${ }^{57}$ These aggregates can then disassemble on binding to a receptor producing fluorogenic response. In our studies, amphiphilic probe NR12S displayed much lower quantum yield in water (0.002) compared to parent Nile Red (0.05) with characteristic blue shifted absorption, suggesting formation of non-fluorescent micellar $\mathrm{H}$-aggregates. ${ }^{16}$ On binding to lipid membranes these aggregates disassembled into molecular species exhibiting high quantum yield $(\sim 0.5)$. Then, we synthesized an analogue of NR12S that was polymerized with crosslinker bearing S-S bond.58 The obtained polymerized micelles were non-fluorescent in aqueous media, but disassembled under reductive stimuli producing desired fluorescence turn-on. Thus, we obtained a fluorogenic membrane probe activatable by a reductive stimulus. Later on, we extended the ACQ approach to squaraine-based probe dSQ12S, displaying $>100$-fold fluorescence turn-on on binding to lipid membranes. ${ }^{59}$ Due to high brightness $\left(\varepsilon \sim 350,000 \mathrm{M}^{-1} \mathrm{~cm}^{-1}\right.$ and quantum yield $\left.\sim 0.5\right)$ and efficient membrane staining, it enabled imaging cell plasma membranes at $1 \mathrm{nM}$ concentration, which is $\sim 1000$-fold lower than needed for conventional membrane probe DiD. The aggregation of dyes does not always lead to ACQ. It can also produce fluorescence enhancement, especially for molecular rotors of propeller-like topology (e.g. tetraphenylethene, hexaphenylsilole, etc). This phenomenon, socalled Aggregation-Induced emission (AIE), has been a subject of intensive research in the last decade. ${ }^{60}$ The design of environment-sensitive probes for biosensing based on AIE concept emerged in the last years, with applications to detection of proteins, nucleic acids, polysacahrides, etc. ${ }^{61}$

(a)

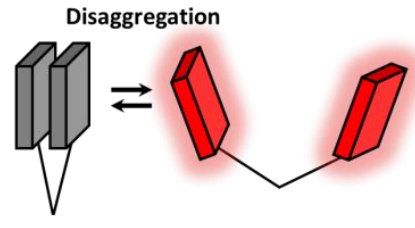

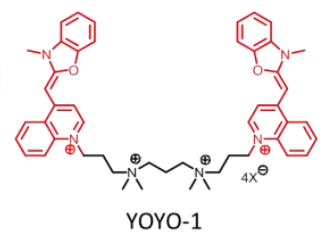

YOYO-1 (b)

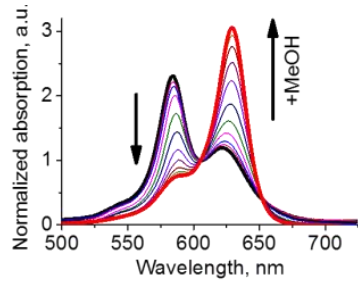

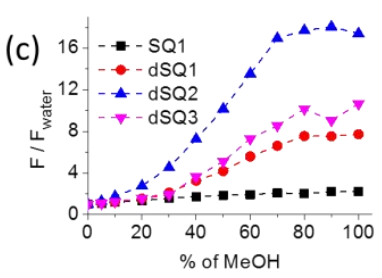
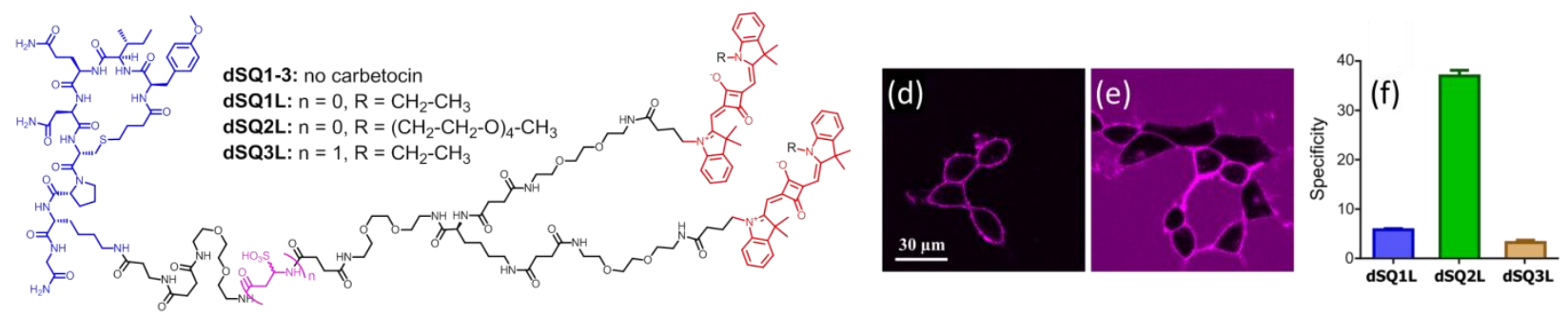

Fig. 9. Probes operating by aggregation-caused quenching mechanism. (a) Principle and examples of these probes. (b) Absorption of squaraine dimer dSQ1 in water with addition of methanol (from 0 till 100\%). (c) Fluorescence intensities (F/Fwater) of dyes in $\mathrm{H} 2 \mathrm{O}-\mathrm{MeOH}$ mixtures with respect to their intensity in water. (d,e) Confocal images of HEK cells expressing the oxytocin receptor with $100 \mathrm{nM}$ of dSQ2L (d) or rhodamine-carbetocin conjugate (e). (f) Specificity of the probes $(10 \mathrm{nM})$ : intensity ratio before and after addition of nonlabeled carbetocin $(2 \mu \mathrm{M})$. Reprinted with permission from ref. ${ }^{55}$. Copyright 2015 American Chemical Society.

\section{Quantitative and background-free bioimaging with environment-sensitive probes}

Fluorescence imaging is usually based on analysis of fluorescence intensity, which depends on many factors, such as probe concentration, light source power, sensitivity of the detector, sampling time, etc. Therefore, quantitative 
fluorescence microscopy uses ratiometric and lifetime imaging, independent on the instrumental settings mentioned above. FLIM and ratio imaging require different type of fluorescent probes. Solvatochromic push-pull and ESIPT dyes perfectly suit to ratiometric imaging, because they change their emission color that can be directly quantified as the ratio at the two channels (Fig. 10a,b). ${ }^{8}$ On the other hand, fluorogenic probes, especially molecular rotors, are more appropriate for lifetime imaging. ${ }^{41}$ Nevertheless, many solvatochromic dyes can change their lifetime in response to polarity and thus can be used for FLIM. Moreover, as we showed for PA probe, FLIM at the blue edge of the emission band can be used even for solvatochromic dyes that do not change much their lifetime (Fig. 10c-e). ${ }^{8}$
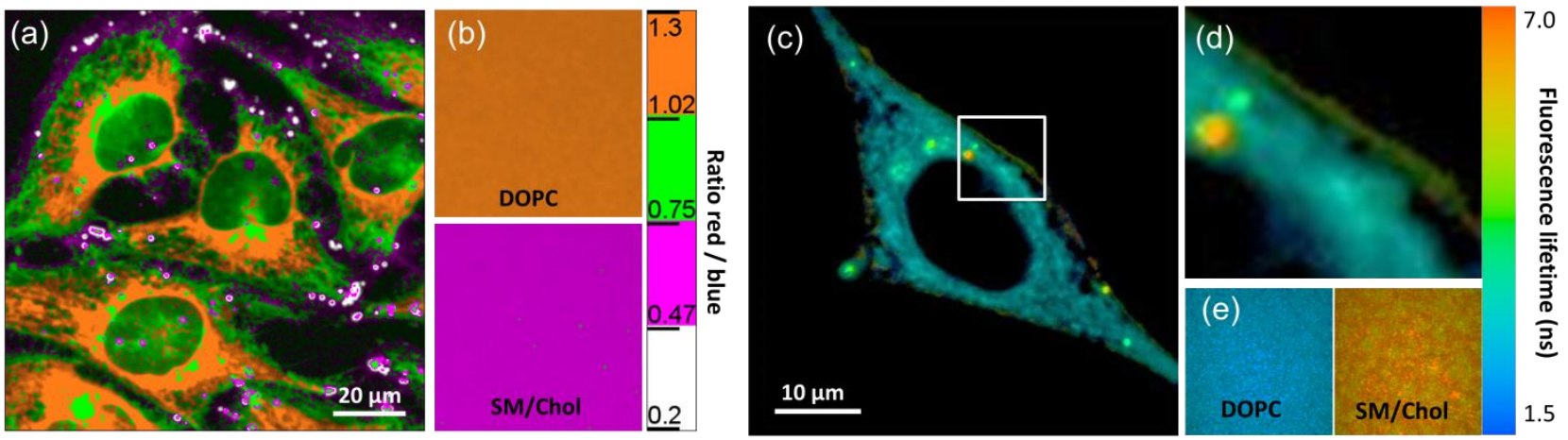

Fig. 10. Ratiometric (a-b) and FLIM (c-e) imaging of lipid order in living HeLa cells using solvatochromic probe PA. The pseudo-colors represent the ratio of the long- to short-wavelength emission channels (550-700 nm to 470-550 $\mathrm{nm}$ ). (b) Calibration of the ratio using suspension of lipid vesicles composed of DOPC (Ld phase) and sphingomyelin/cholesterol (SM/Cho, Lo phase). (c) FLIM image of HeLa cells and (d) zoom on the region of interest. Calibration images of suspensions of DOPC and SM/Chol (e) vesicles recorded with the same instrumental settings. Adapted with permission from ref. ${ }^{8}$. Copyright 2016 Nature Publishing Group.

The second aspect is background-free imaging. The fluorogenic dyes can significantly decrease the background signal, especially at high probe concentrations. Our comparative study of turn-on probe with a rhodamine derivative suggested that fluorogenic response become important starting from $100 \mathrm{nM}$ ligand concentration (Fig. 9d,e). 25,55 Fluorogenic response is also crucial for specific labeling of biomolecules inside the cells, ${ }^{51,52}$ because the fluorescent label, being used in excess, can be a source of strong background. Moreover, fluorogenic response can improve imaging contrast in different superresolution techniques, including stimulated emission depletion (STED), SIM and Stochastic optical reconstruction microscopy (STORM). ${ }^{62}$ Finally, point accumulation for imaging in nanoscale topography (PAINT) exploits directly fluorogenic and chromogenic response of dyes (e.g. Nile Red) to obtain superresolution image and decipher co-existence of different probe environments (e.g. lipid domains). ${ }^{63}$

\section{Conclusions and outlook}

Due to sensitivity to fundamental properties of the environment, such as polarity, viscosity and molecular order, fluorogenic and chromogenic probes can help understanding properties of lipid membranes, monitoring interactions of biomolecules, imaging target biomolecules directly in living cells with minimal background. Further development of environment-sensitive probes should address some remaining problems. First, nonspecific interactions should be minimized to prevent false-positive response of these probes, which can be achieved by, for example, PEGylation or the use of highly specific water-soluble ligands. ${ }^{25,55}$ Second, fluorescence brightness and photostability of push-pull, ESIPT and molecular rotor dyes is generally inferior compared to classical dyes, such as cyanines, rhodamines and BODIPYs. Recent examples, using large pyrene moiety ${ }^{8}$ and boron-based bridge 45 in the push-pull dyes, provide some clues to address these issues. Moreover, emerging in vivo applications will require further shifting of the absorption and emission wavelengths of these dyes to NIR region. In case of ACQ-based dyes, it could be realized 
using conventional NIR dyes, whereas for others, a dedicated design of fluorophores with extended conjugation will be required. The use of fluorogenic and chromogenic probes in lifetime and ratiometric imaging can already provide quantitative information. Moreover, the capacity of fluorogenic probes to increase the signal-to-background ratio is a key to enhance rapidly developing superresolution microscopy. Many new biological applications remain to be discovered for the environment-sensitive dyes and we expect them to become universal tools in the development of next generation of biosensors and probes that can enhance quality and information content of bioimaging.

\section{Biographical Information}

Andrey Klymchenko was born in Kherson, Ukraine, in 1976. He has received his PhD degree in 2003 from Kiev Taras Shevchenko University. After post-doctoral work at the University of Strasbourg and the Catholic University of Leuven, he joined CNRS at the University of Strasbourg in 2006. He got promoted to CNRS Research Director in 2014 and he is an ERC Consolidator fellow since 2015. He is a leader of "Nanochemistry and Bioimaging" group. His research interests include functional fluorescent molecules and nanomaterials for biosensing, imaging and theranostics.

\section{Acknowledgements}

I would like to thank all collaborators listed in joint publications. Jurga Valanciunaite and Iuliia Karpenko are acknowledged for reading the manuscript and fruitful suggestions. This work was supported by the European Research council (ERC) Consolidator grant BrightSens 648528.

\section{References}

(1) Santos-Figueroa, L. E.; Moragues, M. E.; Climent, E.; Agostini, A.; Martínez-Máñez, R.; Sancenón, F.: Chromogenic and fluorogenic chemosensors and reagents for anions. A comprehensive review of the years 20102011. Chem. Soc. Rev. 2013, 42, 3489-3613.

(2) Han, J. Y.; Burgess, K.: Fluorescent Indicators for Intracellular pH. Chem. Rev. 2010, 110, 27092728.

(3) Nadler, A.; Schultz, C.: The power of fluorogenic probes. Angew. Chem. Int. Ed. 2013, 52, 2408-2410.

(4) Zhu, H.; Fan, J. L.; Du, J. J.; Peng, X. J.: Fluorescent Probes for Sensing and Imaging within Specific Cellular Organelles. Acc. Chem. Res. 2016, 49, 2115-2126.

(5) Li, X.; Gao, X.; Shi, W.; Ma, H.: Design strategies for water-soluble small molecular chromogenic and fluorogenic probes. Chem. Rev. 2014, 114, 590-659.

(6) Kucherak, O. A.; Didier, P.; Mèly, Y.; Klymchenko, A. S.: Fluorene analogues of prodan with superior fluorescence brightness and solvatochromism. J. Phys. Chem. Lett. 2010, 1, 616-620.

(7) Niko, Y.; Kawauchi, S.; Konishi, G.: Solvatochromic Pyrene Analogues of Prodan Exhibiting Extremely High Fluorescence Quantum Yields in Apolar and Polar Solvents. Chem. Eur. J. 2013, 19, 9760-9765.

(8) Niko, Y.; Didier, P.; Mely, Y.; Konishi, G.; Klymchenko, A. S.: Bright and photostable push-pull pyrene dye visualizes lipid order variation between plasma and intracellular membranes. Sci. Rep. 2016, 6, 18870.

(9) Lu, Z. K.; Liu, N.; Lord, S. J.; Bunge, S. D.; Moerner, W. E.; Twieg, R. J.: Bright, Red SingleMolecule Emitters: Synthesis and Properties of Environmentally Sensitive Dicyanomethylenedihydrofuran (DCDHF) Fluorophores with Bisaromatic Conjugation. Chem. Mater. 2009, 21, 797-810.

(10) Grabowski, Z. R.; Rotkiewicz, K.; Rettig, W.: Structural Changes Accompanying Intramolecular Electron Transfer: Focus on Twisted Intramolecular Charge-Transfer States and Structures. Chem. Rev. 2003, 103, 3899-4031.

(11) Demchenko, A. P.; Mély, Y.; Duportail, G.; Klymchenko, A. S.: Monitoring biophysical properties of lipid membranes by environment-sensitive fluorescent probes. Biophys. J. 2009, 96, 3461-3470.

(12) Lingwood, D.; Simons, K.: Lipid rafts as a membrane-organizing principle. Science 2010, 327, 46-50.

(13) Klymchenko, A. S.; Kreder, R.: Fluorescent Probes for Lipid Rafts: From Model Membranes to Living Cells. Chem. Biol. 2014, 21, 97-113. 
(14) Bagatolli, L. A.: To see or not to see: Lateral organization of biological membranes and fluorescence microscopy. Biochim. Biophys. Acta 2006, 1758, 1541-1556.

(15) Jin, L.; Millard, A. C.; Wuskell, J. P.; Dong, X. M.; Wu, D. Q.; Clark, H. A.; Loew, L. M.: Characterization and application of a new optical probe for membrane lipid domains. Biophysical J. 2006, 90, 25632575 .

(16) Kucherak, O. A.; Oncul, S.; Darwich, Z.; Yushchenko, D. A.; Arntz, Y.; Didier, P.; Mély, Y.; Klymchenko, A. S.: Switchable nile red-based probe for cholesterol and lipid order at the outer leaflet of biomembranes. J. Am. Chem. Soc. 2010, 132, 4907-4916.

(17) Darwich, Z.; Klymchenko, A. S.; Kucherak, O. A.; Richert, L.; Mély, Y.: Detection of apoptosis through the lipid order of the outer plasma membrane leaflet. Biochim. Biophys. Acta 2012, 1818, 3048-3054.

(18) Darwich, Z.; Klymchenko, A. S.; Dujardin, D.; Mély, Y.: Imaging lipid order changes in endosome membranes of live cells by using a Nile Red-based membrane probe. RSC Adv. 2014, 4, 8481-8488.

(19) Ziomkiewicz, I.; Loman, A.; Klement, R.; Fritsch, C.; Klymchenko, A. S.; Bunt, G.; Jovin, T. M.; Arndt-Jovin, D. J.: Dynamic conformational transitions of the EGF receptor in living mammalian cells determined by FRET and fluorescence lifetime imaging microscopy. Cytometry $A$ 2013, 83, 794-805.

(20) Neuberg, P.; Perino, A.; Morin-Picardat, E.; Anton, N.; Darwich, Z.; Weltin, D.; Mely, Y.; Klymchenko, A. S.; Remy, J. S.; Wagner, A.: Photopolymerized micelles of diacetylene amphiphile: physical characterization and cell delivery properties. Chem. Commun. 2015, 51, 11595-11598.

(21) Montana, V.; Farkas, D. L.; Loew, L. M.: Dual-wavelength ratiometric fluorescence measurements of membrane-potential. Biochemistry 1989, 28, 4536-4539.

(22) Klymchenko, A. S.; Mely, Y.: Fluorescent environment-sensitive dyes as reporters of biomolecular interactions. Prog. Mol. Biol. Transl. Sci. 2013, 113, 35-58.

(23) Cohen, B. E.; McAnaney, T. B.; Park, E. S.; Jan, Y. N.; Boxer, S. G.; Jan, L. Y.: Probing protein electrostatics with a synthetic fluorescent amino acid. Science 2002, 296, 1700-1703.

(24) Venkatraman, P.; Nguyen, T. T.; Sainlos, M.; Bilsel, O.; Chitta, S.; Imperiali, B.; Stern, L. J.: Fluorogenic probes for monitoring peptide binding to class II MHC proteins in living cells. Nat. Chem. Biol. 2007, 3 , 222-228.

(25) Karpenko, I. A.; Kreder, R.; Valencia, C.; Villa, P.; Mendre, C.; Mouillac, B.; Mély, Y.; Hibert, M.; Bonnet, D.; Klymchenko, A. S.: Red fluorescent turn-on ligands for imaging and quantifying $G$ protein-coupled receptors in living cells. ChemBioChem 2014, 15, 359-363.

(26) Demchenko, A. P.; Tang, K. C.; Chou, P. T.: Excited-state proton coupled charge transfer modulated by molecular structure and media polarization. Chem. Soc. Rev. 2013, 42, 1379-1408.

(27) Yushchenko, D. A.; Shvadchak, V. V.; Klymchenko, A. S.; Duportail, G.; Mely, Y.; Pivovarenko, V. G.: 2-Aryl-3-hydroxyquinolones, a new class of dyes with solvent dependent dual emission due to excited state intramolecular proton transfer. New J. Chem. 2006, 30, 774-781.

(28) Klymchenko, A. S.; Pivovarenko, V. G.; Ozturk, T.; Demchenko, A. P.: Modulation of the solventdependent dual emission in 3-hydroxychromones by substituents. New J. Chem. 2003, 27, 1336-1343.

(29) Klymchenko, A. S.; Demchenko, A. P.: Multiparametric probing of intermolecular interactions with fluorescent dye exhibiting excited state intramolecular proton transfer. Phys. Chem. Chem. Phys. 2003, 5, 461-468.

(30) Shynkar, V. V.; Klymchenko, A. S.; Duportail, G.; Demchenko, A. P.; Mely, Y.: Two-color fluorescent probes for imaging the dipole potential of cell plasma membranes. Biochim. Biophys. Acta 2005, 1712, 128136.

(31) Klymchenko, A. S.; Stoeckel, H.; Takeda, K.; Mely, Y.: Fluorescent probe based on intramolecular proton transfer for fast ratiometric measurement of cellular transmembrane potential. J. Phys. Chem. B 2006, 110, 13624-13632.

(32) Klymchenko, A. S.; Oncul, S.; Didier, P.; Schaub, E.; Bagatolli, L.; Duportail, G.; Mély, Y.: Visualization of lipid domains in giant unilamellar vesicles using an environment-sensitive membrane probe based on 3-hydroxyflavone. Biochim. Biophys. Acta 2009, 1788, 495-499.

(33) Shynkar, V. V.; Klymchenko, A. S.; Kunzelmann, C.; Duportail, G.; Muller, C. D.; Demchenko, A. P.; Freyssinet, J. M.; Mely, Y.: Fluorescent biomembrane probe for ratiometric detection of apoptosis. J. Am. Chem. Soc. 2007, 129, 2187-2193.

(34) Strizhak, A. V.; Postupalenko, V. Y.; Shvadchak, V. V.; Morellet, N.; Guittet, E.; Pivovarenko, V. G.; Klymchenko, A. S.; Mély, Y.: Two-color fluorescent L-amino acid mimic of tryptophan for probing peptidenucleic acid complexes. Bioconjugate Chem. 2012, 23, 2434-2443. 
(35) Enander, K.; Choulier, L.; Olsson, A. L.; Yushchenko, D. A.; Kanmert, D.; Klymchenko, A. S.; Demchenko, A. P.; Mély, Y.; Altschuh, D.: A peptide-based, ratiometric biosensor construct for direct fluorescence detection of a protein analyte. Bioconjugate Chem. 2008, 19, 1864-1870.

(36) Postupalenko, V. Y.; Zamotaiev, O. M.; Shvadchak, V. V.; Strizhak, A. V.; Pivovarenko, V. G.; Klymchenko, A. S.; Mely, Y.: Dual-fluorescence 1 -amino acid reports insertion and orientation of melittin peptide in cell membranes. Bioconjug. Chem. 2013, 24, 1998-2007.

(37) Dziuba, D.; Postupalenko, V. Y.; Spadafora, M.; Klymchenko, A. S.; Guérineau, V.; Mély, Y.; Benhida, R.; Burger, A.: A universal nucleoside with strong two-band switchable fluorescence and sensitivity to the environment for investigating DNA interactions. J. Am. Chem. Soc. 2012, 134, 10209-10213.

(38) Yushchenko, D. A.; Fauerbach, J. A.; Thirunavukkuarasu, S.; Jares-Erijman, E. A.; Jovin, T. M.: Fluorescent Ratiometric MFC Probe Sensitive to Early Stages of alpha-Synuclein Aggregation. J. Am. Chem. Soc. 2010, 132, 7860-7861.

(39) Zamotaiev, O. M.; Postupalenko, V. Y.; Shvadchak, V. V.; Pivovarenko, V. G.; Klymchenko, A. S.; Mély, Y.: Monitoring penetratin interactions with lipid membranes and cell internalization using a new hydrationsensitive fluorescent probe. Org. Biomol. Chem. 2014, 12, 7036-7044.

(40) Haidekker, M. A.; Theodorakis, E. A.: Molecular rotors - Fluorescent biosensors for viscosity and flow. Org. Biomol. Chem. 2007, 5, 1669-1678.

(41) Kuimova, M. K.; Yahioglu, G.; Levitt, J. A.; Suhling, K.: Molecular rotor measures viscosity of live cells via fluorescence lifetime imaging. J. Am. Chem. Soc. 2008, 130, 6672-6673.

(42) Amdursky, N.; Erez, Y.; Huppert, D.: Molecular Rotors: What Lies Behind the High Sensitivity of the Thioflavin-T Fluorescent Marker. Acc. Chem. Res. 2012, 45, 1548-1557.

(43) Paige, J. S.; Wu, K. Y.; Jaffrey, S. R.: RNA Mimics of Green Fluorescent Protein. Science 2011, 333 ,

(44) Walker, C. L.; Lukyanov, K. A.; Yampolsky, I. V.; Mishin, A. S.; Bommarius, A. S.; Duraj-Thatte, A. M.; Azizi, B.; Tolbert, L. M.; Solntsev, K. M.: Fluorescence imaging using synthetic GFP chromophores. Curr. Opin. Chem. Biol. 2015, 27, 64-74.

(45) Karpenko, I. A.; Niko, Y.; Yakubovskyi, V. P.; Gerasov, A. O.; Bonnet, D.; Kovtun, Y. P.; Klymchenko, A. S.: Push-pull dioxaborine as fluorescent molecular rotor: far-red fluorogenic probe for ligandreceptor interactions. J. Mater. Chem. C 2016, 4, 3002-3009.

(46) Kuimova, M. K.; Botchway, S. W.; Parker, A. W.; Balaz, M.; Collins, H. A.; Anderson, H. L.; Suhling, K.; Ogilby, P. R.: Imaging intracellular viscosity of a single cell during photoinduced cell death. Nat. Chem. 2009, 1, 69-73.

(47) Telmer, C. A.; Verma, R.; Teng, H. B.; Andreko, S.; Law, L.; Bruchez, M. P.: Rapid, Specific, Nowash, Far-red Fluorogen Activation in Subcellular Compartments by Targeted Fluorogen Activating Proteins. ACS Chem. Biol. 2015, 10, 1239-1246.

(48) Lopez-Duarte, I.; Vu, T. T.; Izquierdo, M. A.; Bull, J. A.; Kuimova, M. K.: A molecular rotor for measuring viscosity in plasma membranes of live cells. Chem. Commun. 2014, 50, 5282-5284.

(49) Dal Molin, M.; Verolet, Q.; Colom, A.; Letrun, R.; Derivery, E.; Gonzalez-Gaitan, M.; Vauthey, E.; Roux, A.; Sakai, N.; Matile, S.: Fluorescent Flippers for Mechanosensitive Membrane Probes. J. Am. Chem. Soc. 2015, $137,568-571$.

(50) Hwan, M. K.; Byeong, H. J.; Hyon, J. Y.; Myoung, J. A.; Mun, S. S.; Jin, H. H.; Lee, K. J.; Chul, H. K.; Joo, T.; Hong, S. C.; Bong, R. C.: Two-photon fluorescent turn-on probe for lipid rafts in live cell and tissue. J. Am. Chem. Soc. 2008, 130, 4246-4247.

(51) Lukinavicius, G.; Umezawa, K.; Olivier, N.; Honigmann, A.; Yang, G. Y.; Plass, T.; Mueller, V.; Reymond, L.; Correa, I. R.; Luo, Z. G.; Schultz, C.; Lemke, E. A.; Heppenstall, P.; Eggeling, C.; Manley, S.; Johnsson, K.: A near-infrared fluorophore for live-cell super-resolution microscopy of cellular proteins. Nat. Chem. 2013, 5, 132-139.

(52) Lukinavicius, G.; Reymond, L.; Umezawa, K.; Sallin, O.; D'Este, E.; Gottfert, F.; Ta, H.; Hell, S. W.; Urano, Y.; Johnsson, K.: Fluorogenic Probes for Multicolor Imaging in Living Cells. J. Am. Chem. Soc. 2016, 138, 9365-9368.

(53) Wurthner, F.; Kaiser, T. E.; Saha-Moller, C. R.: J-Aggregates: From Serendipitous Discovery to Supramolecular Engineering of Functional Dye Materials. Angew. Chem. Int. Ed. 2011, 50, 3376-3410.

(54) Okamoto, A.: ECHO probes: a concept of fluorescence control for practical nucleic acid sensing. Chem. Soc. Rev. 2011, 40, 5815-5828. 
(55) Karpenko, I. A.; Collot, M.; Richert, L.; Valencia, C.; Villa, P.; Mely, Y.; Hibert, M.; Bonnet, D.; Klymchenko, A. S.: Fluorogenic Squaraine Dimers with Polarity-Sensitive Folding As Bright Far-Red Probes for Background-Free Bioimaging. J. Am. Chem. Soc. 2015, 137, 405-412.

(56) Yao, D.; Lin, Z.; Wu, J.: Near-Infrared Fluorogenic Probes with Polarity-Sensitive Emission for in Vivo Imaging of an Ovarian Cancer Biomarker. ACS Appl. Mater. Interfaces 2016, 8, 5847-5856.

(57) Mizusawa, K.; Takaoka, Y.; Hamachi, I.: Specific cell surface protein imaging by extended selfassembling fluorescent turn-on nanoprobes. J. Am. Chem. Soc. 2012, 134, 13386-13395.

(58) Niko, Y.; Arntz, Y.; Mely, Y.; Konishi, G.-I.; Klymchenko, A. S.: Disassembly-Driven Fluorescence Turn-on of Polymerized Micelles by Reductive Stimuli in Living Cells. Eur. J. Chem. 2014, 20, 16473-16477.

(59) Collot, M.; Kreder, R.; Tatarets, A. L.; Patsenker, L. D.; Mely, Y.; Klymchenko, A. S.: Bright fluorogenic squaraines with tuned cell entry for selective imaging of plasma membrane vs. endoplasmic reticulum. Chem. Commun. 2015, 51, 17136-17139.

(60) Mei, J.; Leung, N. L. C.; Kwok, R. T. K.; Lam, J. W. Y.; Tang, B. Z.: Aggregation-Induced Emission: Together We Shine, United We Soar! Chem. Rev. 2015, 115, 11718-11940.

(61) Kwok, R. T. K.; Leung, C. W. T.; Lam, J. W. Y.; Tang, B. Z.: Biosensing by luminogens with aggregation-induced emission characteristics. Chem. Soc. Rev. 2015, 44, 4228-4238.

(62) Schermelleh, L.; Heintzmann, R.; Leonhardt, H.: A guide to super-resolution fluorescence microscopy. J. Cell Biol. 2010, 190, 165-175.

(63) Bongiovanni, M. N.; Godet, J.; Horrocks, M. H.; Tosatto, L.; Carr, A. R.; Wirthensohn, D. C.; Ranasinghe, R. T.; Lee, J. E.; Ponjavic, A.; Fritz, J. V.; Dobson, C. M.; Klenerman, D.; Lee, S. F.: Multi-dimensional super-resolution imaging enables surface hydrophobicity mapping. Nat. Commun. 2016, 7, 13544. 